\title{
Comparative survey of go/no-go results to identify the inhibitory control ability change of Japanese children
}

\author{
Koji Terasawa ${ }^{1 *}$, Hisaaki Tabuchi ${ }^{1}$, Hiroki Yanagisawa ${ }^{2}$, Akitaka Yanagisawa $^{3}$, Kikunori Shinohara ${ }^{4}$, Saiki Terasawa ${ }^{5}$, \\ Osamitsu Saijo ${ }^{6}$ and Takeo Masaki ${ }^{6}$
}

\begin{abstract}
This research, conducted in 1998 and 2008, uses go/no-go data to investigate the fundamentals of cognitive functioning in the inhibitory control ability of Japanese children. 844 subjects from kindergarten to junior high school participated in go/no-go task experiments. Performance of go/no-go tasks, which are frequently used to investigate response inhibition, measures a variety of cognitive components besides response inhibition. With normal brain development, the ability to inhibit responses improves substantially in adolescence. An increase over time in the error rate during the go/no-go tasks of subjects of the same age indicates that these processes are not functioning properly. Comparisons between the 1998 and 2008 data revealed several differences in error rates. In 2008, there were increases in the number of errors in groups from each age range. The comparison also revealed that overall error rates peaked at later ages in the 2008 subjects. Taken together, these results show changing conditions in the inhibitory function of the prefrontal cortex. However, the reason for these changing conditions remains unclear. While a lifestyle questionnaire revealed several differences in factors such as bedtimes and hours spent watching TV, analysis did not reveal a significant correlation.
\end{abstract}

\section{Introduction}

Cognitive control functions continue to improve from infancy until early adulthood, allowing flexible adaptation to a complex environment [1]. Growth in executive functioning skills play a role in children's academic success, and the transition to elementary school is an important time for the development of these abilities [2]. Executive functions make it possible to mentally play with ideas and take the time to think before acting. Core executive functions are inhibition and interference control, working memory, and cognitive flexibility [3]. Both inhibitory-based executive functioning and basic information processing deficits are found in clinic-referred attention deficit hyperactivity disorder samples [4].

Response inhibition is an essential executive function implemented by the prefrontal cortex.

\footnotetext{
*Correspondence: kterasa@shinshu-u.ac.jp

${ }^{1}$ Shinshu University, Faculty of Education, 6-Ro Nishinagano Naganoshi, Nagano 380-8544, Japan

Full list of author information is available at the end of the article
}

Recent neuropsychological and neuroimaging studies have shown that the presupplementary motor area and ventrolateral prefrontal cortex are crucial for response inhibition and that various subregions of the prefrontal cortex make different contributions leading to successful response inhibition [5]. Performance of go/no-go tasks, which are frequently used to investigate response inhibition, requires a variety of cognitive components besides response inhibition [6].

In view of the changing cultural conditions and their potential influence on response inhibitory function implemented by the prefrontal cortex, we have been investigating the condition of children's inhibitory function. In particular, we have been focusing on the inhibitory control ability using go/no-go tasks since 1969 [7-10]. The number of errors made by sixth and seventh graders in go/no-go tasks has been increasing since around 1980.

Despite this, no research has applied go/no-go tasks to subjects from kindergarten to junior high school to explore the process of inhibitory development. Thus, we 
investigated the developmental inhibitory process of the repression function of children from kindergarten to junior high school using go/no-go tasks.

\section{Methods}

\section{Participants}

The participants were children aged 3 to 15 years from the same kindergarten-to-junior high school (G1 to G12). 437 children (225 boys and 212 girls) participated in 1998 and 407 different children (200 boys and 207 girls) participated in 2008 (see Table 1 for details). In this investigation, no children with ADHD or autism were included. The latest guidelines of the Helsinki Declaration were followed, and the study was approved by the Institutional Ethics Committee of Shinshu University. Written informed consent was obtained from the children and their parents.

\section{Task}

The go/no-go task consisted of the following three stages: formation, differentiation, and reverse differentiation. In the formation stage, the participants were trained to squeeze a rubber ball in response to a red light stimulus, which was displayed five times (Figure 1A). For the differentiation stage, they were asked to squeeze it in response to red but not to yellow (Figure 1B). For the reverse differentiation stage, the instructions were reversed; that is, they squeezed the ball in response to yellow but not to red. Each participant performed 20 trials each during the differentiation and reverse differentiation stages and the number of errors from the 40 trials of the two stages was calculated. Yellow and red stimuli were used for G1 to G9 subjects, and bright and dim lights were used as stimuli for G10 to G12 subjects. The duration of each stimulus was random between 200 and $1100 \mathrm{~ms}$. The inter-stimulus interval was also random between 1300 and $7500 \mathrm{~ms}$.

Table 1 The number of participants

\begin{tabular}{cccc}
\hline Grade level & Age (years) & $\mathbf{1 9 9 8}$ & $\mathbf{2 0 0 8}$ \\
\hline G1 & $3-4$ & 23 & 28 \\
\hline G2 & $4-5$ & 53 & 46 \\
\hline G3 & $5-6$ & 53 & 30 \\
\hline G4 & $6-7$ & 37 & 33 \\
\hline G5 & $7-8$ & 37 & 35 \\
\hline G6 & $8-9$ & 32 & 28 \\
\hline G7 & $9-10$ & 35 & 29 \\
\hline G8 & $10-11$ & 33 & 35 \\
\hline G9 & $11-12$ & 32 & 31 \\
\hline G10 & $12-13$ & 36 & 38 \\
\hline G11 & $13-14$ & 31 & 38 \\
\hline G12 & $14-15$ & 35 & 36 \\
\hline
\end{tabular}

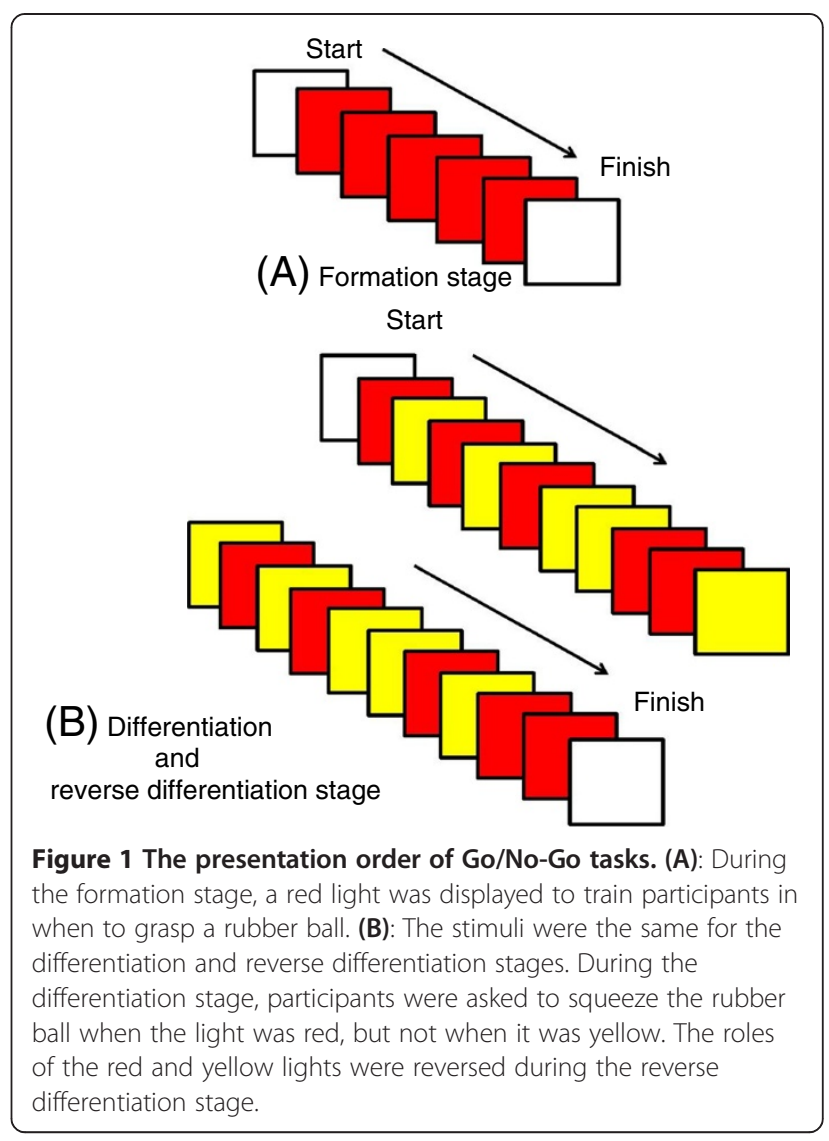

The participants were seated in a booth enclosed on three sides by $45 \times 45 \mathrm{~cm}$ panels. Stimuli were presented in a framed rectangle $(2.5 \mathrm{~cm} \times 3.7 \mathrm{~cm})$, which was approximately $70 \mathrm{~cm}$ away from their eyes. The experiment was conducted by computer-controlled equipment (ME Corporation, Nagano, Japan).

\section{Statistical analysis}

We used the number of errors for statistical analysis of the go/no-go tasks. Reaction times could not be determined because we used analog data collection in 1998 . In this study, we analyzed the data classified according to age as defined by educational grade. Preliminary analyses were conducted to determine if there were any differences in the number of errors made between each grade in kindergarten, elementary school and junior high school using one-way ANOVA and Tukey's honest significant difference post hoc multiple comparison test. We used two-way ANOVA to make a comparison between 1998 and 2008 data. Because the data obtained from the go/no-go tasks have a limited number of errors and a normal distribution is not assumed in this case, secondary analyses used the Poisson distribution. 
The participant's wakeup time, bedtime, and time spent playing, studying, watching TV and playing videogames were assessed from a questionnaire filled out after the experiment. The results of the questionnaires from 1998 and 2008 were compared using independent $t$-tests. In addition, we investigated the correlation between the number of errors for the go/no-go task and the numerical values of the 2008 questionnaire.

The level of significance was set at $p<0.05$. Statistical analyses were performed using SPSS Statistical Packages (SPSS Inc., Chicago, USA).

\section{Results}

For the convenience of statistical analysis, the participants were categorized into three respective age groups: kindergarten (G1 to G3; ages 3 to 6 years), elementary (G4 to G9; ages 6 to 12 years), and junior high school (G10 to G12; ages 12 to 15 years, see Table 1 for details).

\section{The number of errors in 1998}

Figure 2 shows that the number of errors decreases with age, especially between G1 and G3. An independent ANOVA indicated that the effect of age group was significant $[\mathrm{F}(2,126)=18.7, p<0.001]$. For the kindergarten group, Turkey's HSD analyses showed that the numbers of errors for $\mathrm{G} 2$ (mean $(\mathrm{M})=6.4$, standard error $(\mathrm{SE})=0.9$ ) and $\mathrm{G} 3(\mathrm{M}=4.1, \mathrm{SE}=0.4)$ were significantly lower than for $\mathrm{G} 1(\mathrm{M}=12.0, \mathrm{SE}=1.3)$ [G1 vs. $\mathrm{G} 2, p<0.001$; $\mathrm{G} 1$ vs. $\mathrm{G} 3, p<0.001]$. The results of the elementary-school group from G4 to G9, however, were almost the same across these different ages $[\mathrm{F}(5,200)=1.1, p>0.37]$. In the juniorhigh-school group, the number of errors also decreased with age $[\mathrm{F}(2,99)=27.4, p<0.01]$. The difference between

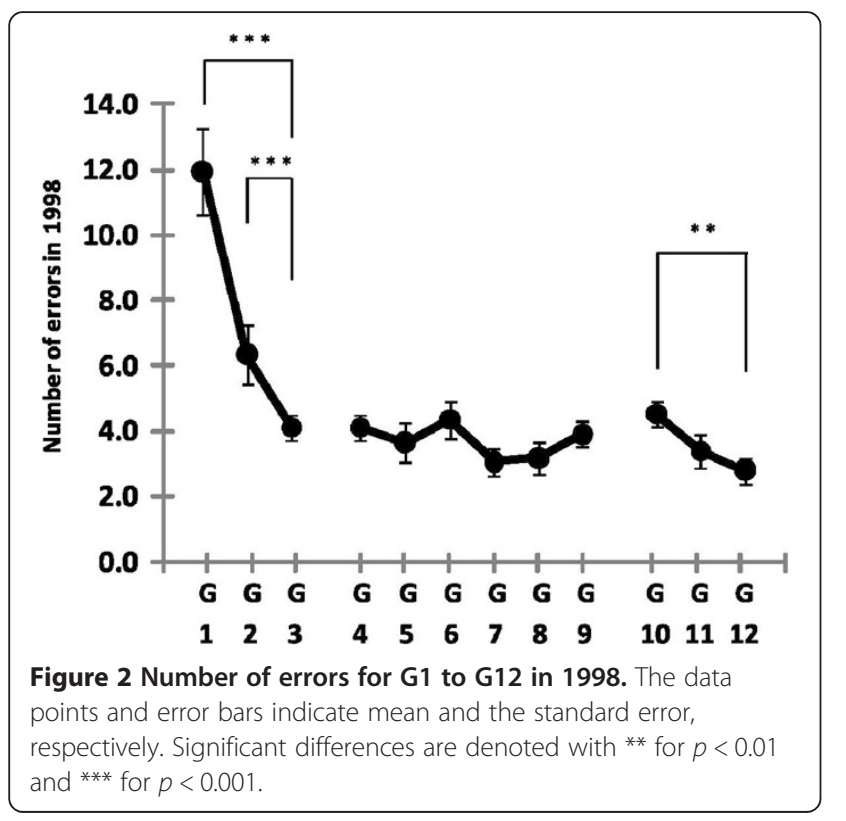

G10 $(\mathrm{M}=4.5, \mathrm{SE}=0.4)$ and $\mathrm{G} 12(\mathrm{M}=2.8, \mathrm{SE}=0.4)$ was significant $[p<0.01]$.

\section{The number of errors in 2008}

Figure 3 shows that the number of errors decreased with age from G1 to G3, similar to what was observed in 1998. An independent ANOVA indicated that the effect of age group was significant $[F(2,101)=24.0, p<0.001]$. The numbers of errors for $\mathrm{G} 2(\mathrm{M}=7.4, \mathrm{SE}=0.5)$ and $\mathrm{G} 3(\mathrm{M}=5.3, \mathrm{SE}=0.5)$ were significantly lower than for $\mathrm{G} 1(\mathrm{M}=12.1, \mathrm{SE}=0.9)$ [G1 vs. $\mathrm{G} 2, \mathrm{p}<0.001 ; \mathrm{G} 1$ vs. $\mathrm{G} 3$, $\mathrm{p}<0.001$ ]. The elementary-school group from G4 to G9 did not show a statistically significant difference in error rate $[F(5,185)=23.5, p>0.09]$. In the junior-high-school group, the difference in the number of errors between G11 $(\mathrm{M}=6.8, \mathrm{SE}=0.7)$ and $\mathrm{G} 12(\mathrm{M}=4.0, \mathrm{SE}=0.6)$ was significant $[\mathrm{p}<0.01]$.

\section{Comparison between 1998 and 2008}

The results of 1998 and 2008 are superimposed in Figure 4. Although the overall patterns are similar, the numbers of errors in 2008 tended to be greater than those of 1998 . The greatest difference for the different years of investigation was found in G11; the average number of erroneous responses was 3.4 in 1998, as opposed to 6.8 in 2008. The effect of the research year was statistically significant $[\mathrm{F}(1,198)=14.7, \mathrm{p}<0.001]$. A post-hoc analysis based on a Poisson distribution indicated that the 2008 results for G5, G8, G10, and G11 were statistically higher than those of 1998 [p $<0.001]$.

\section{Lifestyle survey and correlational investigation}

Table 2 shows the mean response from each survey question in 1998 and 2008. Although some lifestyle

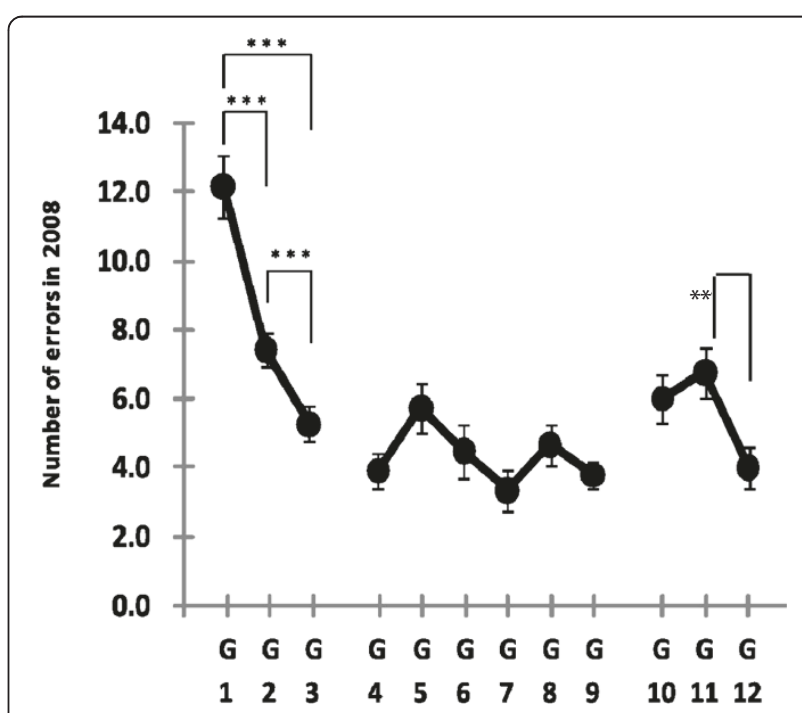

Figure 3 Number of errors for $\mathbf{G 1}$ to $\mathbf{G 1 2}$ in 2008. Significant differences are denoted with ** for $p<0.01$ and *** for $p<0.001$. 


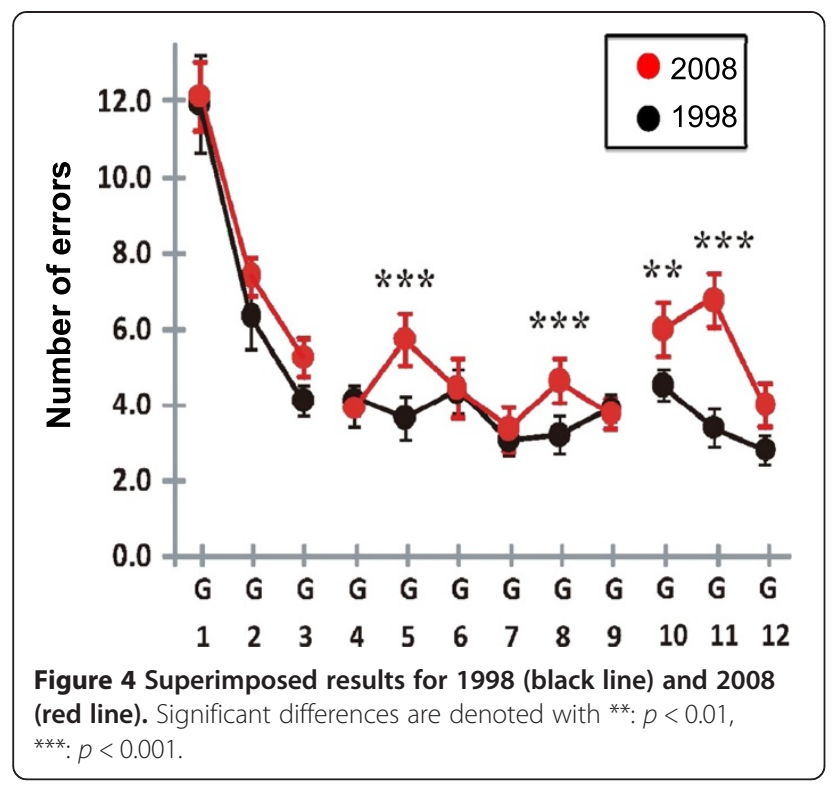

questions, such as wakeup time for G4, bedtime for G5, and time spent studying for G12, were found to be statistically significant between the survey years, the pattern of significance seemed too sporadic to interpret.

Table 3 indicates the correlation between each lifestyle question in 2008 and the number of errors for the go/ no-go task. A positive correlation coefficient indicates that the number of errors increases as the numerical value for the survey question increases, whereas a negative correlation coefficient indicates that the number of errors decreases as the numerical value for the survey question increases. The error rate and time spent watching TV and playing videogames were positively correlated for G5 and G8, although the strength of relationship was moderate or less. A negative correlation was also found for time spent studying for G4 and G8.

\section{Discussion}

Investigations were done in 1998 and 2008 using go/nogo tasks to look into inhibitory control as an essential

Table 2 Results from the lifestyle questionnaire in 1998 and 2008

\begin{tabular}{|c|c|c|c|c|c|c|c|c|c|c|c|c|}
\hline & \multicolumn{4}{|c|}{ G-1 } & \multicolumn{4}{|c|}{ G-2 } & \multicolumn{4}{|c|}{ G-3 } \\
\hline & 1998 & 2008 & $t$ & $p$ & 1998 & 2008 & $t$ & $p$ & 1998 & 2008 & $t$ & $p$ \\
\hline Awake time & 7:07 & $7: 11$ & -0.632 & NS & $7: 16$ & $6: 54$ & 2.587 & $*$ & 7:14 & 7:05 & 1.653 & NS \\
\hline Bedtime & $21: 12$ & $21: 01$ & 0.943 & NS & $21: 08$ & $20: 49$ & 1.873 & NS & 21:09 & $20: 36$ & 1.194 & NS \\
\hline Time to play (min) & 215 & 182 & 1.912 & NS & 227 & 186 & 3.187 & $* *$ & 201 & 155 & 2.790 & ** \\
\hline Time to study (min) & - & - & - & - & - & - & - & - & - & - & - & - \\
\hline \multirow[t]{3}{*}{ Time to watch TV \& play videogame } & 94 & 114 & -1.203 & NS & 101 & 101 & 0.022 & NS & 79 & 87 & -0.487 & NS \\
\hline & \multicolumn{4}{|c|}{ G-4 } & \multicolumn{4}{|c|}{ G-5 } & \multicolumn{4}{|c|}{ G-6 } \\
\hline & 1998 & 2008 & $t$ & $p$ & 1998 & 2008 & $t$ & $p$ & 1998 & 2008 & $t$ & $p$ \\
\hline Awake time & $6: 35$ & $6: 19$ & 3.455 & $* * *$ & $6: 30$ & $6: 17$ & 0.126 & NS & $6: 44$ & $6: 22$ & -1.223 & NS \\
\hline Bedtime & $20: 56$ & $21: 01$ & 0.102 & NS & $20: 54$ & $21: 18$ & -3.491 & $* * *$ & $21: 10$ & $21: 12$ & 1.453 & NS \\
\hline Time to play (min) & 95 & 71 & 2.025 & * & 87 & 73 & 1.156 & NS & 100 & 91 & -1.210 & NS \\
\hline Time to study (min) & 80 & 46 & -0.138 & NS & 115 & 57 & 2.519 & $*$ & 114 & 71 & -0.648 & NS \\
\hline \multirow[t]{3}{*}{ Time to watch TV \& play videogame } & 86 & 83 & 2.077 & $*$ & 133 & 98 & 3.194 & $* *$ & 124 & 174 & -0.871 & NS \\
\hline & \multicolumn{4}{|c|}{ G-7 } & \multicolumn{4}{|c|}{ G-8 } & \multicolumn{4}{|c|}{ G-9 } \\
\hline & 1998 & 2008 & $t$ & $p$ & 1998 & 2008 & $t$ & $p$ & 1998 & 2008 & $t$ & $p$ \\
\hline Awake time & $6: 32$ & $6: 20$ & 0.987 & NS & $6: 32$ & $6: 19$ & -1.408 & NS & $6: 28$ & $6: 19$ & 1.776 & NS \\
\hline Bedtime & $21: 10$ & $21: 32$ & 0.958 & NS & $21: 17$ & $22: 08$ & -2.626 & $* *$ & $21: 32$ & $21: 56$ & -0.398 & NS \\
\hline Time to play (min) & 75 & 75 & -0.120 & NS & 85 & 63 & 0.690 & NS & 60 & 53 & 0.954 & NS \\
\hline Time to study (min) & 158 & 89 & 0.544 & NS & 113 & 98 & -1.498 & NS & 183 & 111 & 0.974 & NS \\
\hline \multirow[t]{3}{*}{ Time to watch TV \& play videogame } & 110 & 132 & 0.395 & NS & 125 & 114 & 1.283 & NS & 93 & 106 & 2.334 & * \\
\hline & \multicolumn{4}{|c|}{ G-10 } & \multicolumn{4}{|c|}{ G-11 } & \multicolumn{4}{|c|}{ G-12 } \\
\hline & 1998 & 2008 & $t$ & $\bar{p}$ & 1998 & 2008 & $t$ & $\bar{p}$ & 1998 & 2008 & $t$ & $p$ \\
\hline Awake time & $5: 45$ & $5: 56$ & -1.336 & NS & $5: 54$ & $5: 57$ & -1.427 & NS & 5:50 & $6: 17$ & -2.593 & * \\
\hline Bedtime & 23:02 & $22: 29$ & 2.790 & $* *$ & $23: 18$ & $23: 17$ & 0.054 & NS & $23: 39$ & $23: 57$ & -1.227 & NS \\
\hline Time to play (min) & 27 & 45 & -1.982 & NS & 67 & 111 & -2.658 & $* *$ & 27 & 132 & -2.922 & $* *$ \\
\hline Time to study (min) & 127 & 119 & 0.592 & NS & 105 & 113 & -0.554 & NS & 218 & 158 & 4.168 & $* * *$ \\
\hline Time to watch TV \& play videogame (min) & 99 & 95 & 0.246 & NS & 117 & 166 & -1.460 & NS & 61 & 97 & -2.804 & ** \\
\hline
\end{tabular}

NS, not significant; ${ }^{*} p<0.05 ;{ }^{* *} p<0.01 ;{ }^{* * *} p<0.001$. 
Table 3 Correlations between number of errors and lifestyle questionnaire responses in 2008

\begin{tabular}{|c|c|c|c|c|c|c|c|c|c|}
\hline & \multicolumn{3}{|c|}{ G-1 } & \multicolumn{3}{|c|}{ G-2 } & \multicolumn{3}{|c|}{ G-3 } \\
\hline & $r$ & $t$ & $p$ & $r$ & $t$ & $p$ & $r$ & $t$ & $p$ \\
\hline Awake time & 0.117 & 0.003 & NS & -0.132 & -0.880 & NS & 0.188 & 0.994 & NS \\
\hline Bedtime & 0.010 & 0.053 & NS & -0.078 & -0.522 & NS & 0.054 & 0.278 & NS \\
\hline Time to play (min) & -0.304 & -1.630 & NS & -0.060 & -0.436 & NS & -0.203 & -1.078 & NS \\
\hline Time to study (min) & - & - & - & - & - & - & - & - & - \\
\hline \multirow[t]{3}{*}{ Time to watch TV \& play videogame (min) } & -0.144 & -0.739 & NS & -0.137 & -0.914 & NS & 0.097 & 0.504 & NS \\
\hline & \multicolumn{3}{|c|}{ G-4 } & \multicolumn{3}{|c|}{ G-5 } & \multicolumn{3}{|c|}{ G-6 } \\
\hline & $r$ & $t$ & $p$ & $r$ & $t$ & $p$ & $r$ & $t$ & $p$ \\
\hline Awake time & -0.140 & -0.746 & NS & -0.121 & -0.692 & NS & 0.297 & 1.583 & NS \\
\hline Bedtime & -0.214 & -1.161 & NS & 0.098 & 0.556 & NS & -0.212 & -1.109 & NS \\
\hline Time to play (min) & 0.032 & 0.172 & NS & -0.024 & -0.139 & NS & 0.198 & 1.027 & NS \\
\hline Time to study (min) & -0.375 & -2.146 & $*$ & -0.306 & -1.821 & NS & 0.015 & 0.077 & NS \\
\hline \multirow[t]{3}{*}{ Time to watch TV \& play videogame (min) } & -0.188 & -1.015 & NS & 0.443 & 2.796 & ** & 0.002 & 0.010 & NS \\
\hline & \multicolumn{3}{|c|}{ G-7 } & \multicolumn{3}{|c|}{ G-8 } & \multicolumn{3}{|c|}{ G-9 } \\
\hline & $r$ & $t$ & $p$ & $r$ & $t$ & $p$ & $r$ & $t$ & $p$ \\
\hline Awake time & 0.149 & 0.737 & NS & 0.045 & 0.256 & NS & 0.039 & 0.212 & NS \\
\hline Bedtime & -0.231 & -1.161 & NS & -0.168 & -0.965 & NS & 0.181 & 0.989 & NS \\
\hline Time to play (min) & 0.183 & 0.911 & NS & 0.049 & 0.277 & NS & -0.072 & -0.391 & NS \\
\hline Time to study (min) & -0.199 & -0.992 & NS & -0.405 & -2.507 & * & 0.225 & 1.243 & NS \\
\hline \multirow[t]{3}{*}{ Time to watch TV \& play videogame (min) } & -0.187 & -0.933 & NS & 0.177 & 1.018 & NS & -0.009 & -0.050 & NS \\
\hline & \multicolumn{3}{|c|}{ G-10 } & \multicolumn{3}{|c|}{ G-11 } & \multicolumn{3}{|c|}{ G-12 } \\
\hline & $r$ & $t$ & $p$ & $r$ & $t$ & $p$ & $r$ & $t$ & $p$ \\
\hline Awake time & 0.058 & 0.346 & NS & -0.051 & -0.308 & NS & -0.030 & -0.172 & NS \\
\hline Bedtime & 0.103 & 0.624 & NS & 0.000 & 0.002 & NS & 0.248 & 1.493 & NS \\
\hline Time to play (min) & 0.022 & 0.129 & NS & 0.118 & 0.724 & NS & 0.337 & 2.090 & * \\
\hline Time to study (min) & 0.127 & 0.769 & NS & 0.137 & 0.842 & NS & -0.182 & -1.080 & NS \\
\hline Time to watch TV \& play videogame (min) & 0.036 & 0.219 & NS & 0.078 & 0.474 & NS & -0.078 & -0.479 & NS \\
\hline
\end{tabular}

r; Correlational coefficient, $t$; $t$ value, $p ; p$ value, NS; not significant; ${ }^{*} \mathrm{p}<0.05$; ${ }^{* *} \mathrm{p}<0.01$.

executive function implemented by the prefrontal cortex in children. The subjects in this study were all ordinary children and the conditions of their academic maturation were the same; the same kindergarten, primary school, and junior high school were used in both years.

The number of errors for kindergarten groups was approximately the same for G1 but greater in 2008 for G2 and G3. The number of errors for primary school groups was virtually the same from G4 to G9. Comparisons between the results from 1998 and 2008 showed no statistically significant differences from G4 to G9. However, a change in the error peak patterns can be seen. The number of errors in 1998 peaks in G6, whereas the 2008 data show a maximum in G5 and a second increase in G8 with both groups showing a statistically significant increase over the same 1998 groups (Figure 3). In 2008, the number of errors was greater in G5 than G3, a pattern that was not observed in 1998. The second peak (G8) in 2008 was not seen previously. These changes indicate that during the 10 years between tests, the inhibitory control ability has declined.

The period from early childhood to school age is important for the development of several brain functions. Dowsett et al. [11] reported that the conceptual understanding of inhibition responses is acquired by the age of 7 in typically developing children.

For junior high school students, in 1998 the number of errors decreased gradually from G10 to G12. However, in 2008, the number peaked in G11. According to the statistical comparisons between 1998 and 2008, the number of errors in G10 and G11 increased significantly in 2008. In addition, the number of errors in G11 increased at a greater rate compared to 1998. This increase suggests 
diminished inhibition control ability in G11. This result shows diminished cognitive components, besides response inhibition, of the brain of Japanese children for the ten years from 1998 to 2008 . We do not yet understand the cause, but it may be some kind of influence such as environmental changes.

Tamm et al. [12] reported that the go/no-go task requires multiple executive functions, including working memory, interference avoidance, and response withholding, which have been established as prepotent responses. Working memory provides for the need to access and manipulate information in the short-term memory system [13]. In recent years, the working memory model has been further supported by neuroimaging studies. Selective attention, the capacity to focus on one stream of information while shutting out irrelevant material, is needed to solve a task immediately [14]. Furthermore, the capacity to switch attention from one source to another is also necessary. These important roles are assumed by working memory. Almost all measures of short-term memory show a steady increase from the preschool years through adolescence: Behavioral measures of working memory systems improve substantially between the ages of 4 and $15[15,16]$. Based on this data, an increased error rate for go/no-go tasks reflects a changed condition in the inhibitory function that is an essential executive function implemented for working memory by the prefrontal cortex.

We carried out a survey to explore possible correlations that may be found in children's lifestyle changes. Significant correlations between the number of errors in the go/no-go tasks and the hours spent watching television and playing videogames, bedtime, and the number of hours of sleep were found in some grades. Overall, however, no significant correlation between the number of errors in the go/no-go tasks and the lifestyle survey was found. From this, we conjecture that the change condition in the inhibitory function is not directly related to lifestyle factors such as the time you, wakeup, bedtime, hours spent studying or hours spent watching television and playing video games. Thus, other potential causes for these delays must be explored.

The Japanese lifestyle survey from 1956 to 1978 reported large time changes on the average [17]. Play -time decreased to 53 minutes, TV watching and listening to stereo and radio increased to 59 minutes, time doing housework decreased to 74 minutes, and studying increased to 104 minutes. This may be related to the decrease in physical activity. In the future, our studies need to include these things.

\section{Competing interests}

The authors declare that they have no competing interests.

\section{Authors' contributions}

$\mathrm{KT}$, OS and TM participated in the design of this study and drafted manuscript. HT, AY, KS and ST carried out data collection and analysed the data. HT provided the information about the psychological variables. ST carried out back translation into English, and confirmed phrasing nuances with HY and KS. OS, TM and HT evaluated the results of the study and reviewed the manuscript. All authors read and approved the final manuscript.

\section{Acknowledgements}

The authors would like to thank Koki Nakajima, Kenichi Nemoto, Tomio Matsumura, Hiroshi Miura, Masao Okuhara, and Satomi Fujimori for technica support.

Koji Terasawa are supported by a Grant-in-Aid for the Scientist (Houga: 26560380, Kiban A: 25257101) of the Ministry of Education, Culture, Sports, Science and Technology of Japan. This study was supported grant of the Preventive Medical Center of Shinshu University Hospital from Ministry of Education, Culture, Science and Technology.

\section{Author details}

'Shinshu University, Faculty of Education, 6-Ro Nishinagano Naganoshi, Nagano 380-8544, Japan. ${ }^{2}$ Physical Fitness Research Institute, Meiji Yasuda Life Foundation of Health and Welfare, 150 Tobukimachi Hachoujisi, Tokyo192-0001, Japan. ${ }^{3}$ Department of Infant Childcare, Matsumoto Junior College, 3118 Sasaga Matsumotosi, Nagano 399-0033, Japan. ${ }^{4}$ Center of General Education and Humanities, Tokyo University of Science, 5000-1 Toyohira Chino, Nagano 391-213, Japan. ${ }^{5}$ Department of Electrical and Electronic Engineering, Shinshu University, 4-17-1 Wakasato Naganoshi, Nagano 380-928, Japan. 'Laboratory of Psychology, Nippon Sport Science University, 7-1-1 Fukasawa Setagayaku, Tokyo 158-0081, Japan.

Received: 19 April 2014 Accepted: 3 July 2014

Published: 11 July 2014

\section{References}

1. Liu ZX, Woltering S, Lewis MD: Developmental change in EEG theta activity in the medial prefrontal cortex during response control. Neuroimage 2014, 2:873-887.

2. Grammer JK, Carrasco M, Gehring WJ, Morrison FJ: Age-related changes in error processing in young children: aschool-based investigation. Developmental Cognitive Neuroscience 2014, 9:93-105.

3. Diamond A: Executive functions. Annu Rev Psychol 2013, 64:135-168.

4. Salum GA, Sergeant J, Sonuga-Barke E, Vandekerckhove J, Gadelha A, Pan PM, Moriyama TS, Graeff-Martins AS, de Alvarenga PG, do Rosário MC, Manfro GG, Polanczyk G, Rohde LA: Specificity of basic information processing and inhibitory control in attention deficit hyperactivity disorder. Psychol Med 2014, 44:617-631.

5. Chikazoe J, Konishi S, Asari T, Jimura K, Miyashita Y: Activation of right inferior frontal gyrus during response inhibition across response modalities. J Cogn Neurosci 2007, 19:69-80.

6. Chikazoe J: Localizing performance of go/no-go tasks to prefrontal cortical subregions. Curr Opin Psychiatry 2010, 23(3):267-272.

7. Masaki T, Moriyama G: A study on the types of the human higher nervous activity. J Tokyo Science Univ 1971, 4:69-81.

8. Saijo O, Mriyama G, Noshi K, Kumano K, Muramoto K, Abe S, Masaki T: A study on the types of the children higher nervous activity. J Nippon Sport Science Univ 1981, 10:61-68.

9. Terasawa K, Zhiyong J: A comparative study: pattern of the higher nervous activity of Chinese and Japanese. J Beijing Institute of Physical Education 1986, 4:87-94. in Chinese.

10. Terasawa K, Saijo O, Shinohara K, Nemoto K, Masaki T, Yanagisawa A: A survey of patterns in the development of cerebral activity in '69, 79, 98 in Japanese and in '84 in Chinese children. J Physiological Anthropology 2000, 5:47-54. in Japanese.

11. Dowsett SM, Livesey DJ: The development of inhibitory control in preschool children: effects of "executive skills" training. Dev Psychobiol 2000, 36:161-174.

12. Tamm L, Menon V, Reiss AL: Maturation of brain function associated with response inhibition. J Am Acad Child Adol Psychiatr 2002, 41:1231-1238 
13. Baddeley AD: Working memory. Oxford: Oxford University Press; 1986.

14. Baddeley AD: Working memory and executive. Philos Trans R Soc Lond B Biol Sci 1996, 29:1397-13403.

15. Gathercole SE, Pickering SJ: Working memory deficits in children with low achievements in the national curriculum at 7 years of age. Br J Educ Psychol 2000, 70(Pt 2):177-94.a.

16. Gathercole SE, Pickering SJ, Ambridge B, Wearing H: The structure of working memory from 4 to 15 years of age. Dev Psychol 2004, 40:177-190.

17. National theater network of children and parents: Culture and environment of the children. Tobi print Limited 1979, 41-60. in Japanese.

doi:10.1186/1751-0759-8-14

Cite this article as: Terasawa et al:: Comparative survey of go/no-go results to identify the inhibitory control ability change of Japanese children. BioPsychoSocial Medicine 2014 8:14

\section{Submit your next manuscript to BioMed Central} and take full advantage of:

- Convenient online submission

- Thorough peer review

- No space constraints or color figure charges

- Immediate publication on acceptance

- Inclusion in PubMed, CAS, Scopus and Google Scholar

- Research which is freely available for redistribution 